\title{
Fractal analysis of the structural complexity of the connective tissue in human carotid bodies
}

\section{Diego Guidolin, Andrea Porzionato*, Cinzia Tortorella, Veronica Macchi and Raffaele De Caro}

Section of Human Anatomy, Department of Molecular Medicine, University of Padova, Padova, Italy

\section{Edited by:}

Rodrigo Iturriaga, Pontificia

Universidad Católica de Chile, Chile

Reviewed by:

Rodrigo Del Rio, Universidad

Autonoma de Chile, Chile

Man Lung Fung, The University of

Hong Kong, China

*Correspondence:

Andrea Porzionato, Section of

Anatomy, Department of Molecular

Medicine, University of Padova, Via

A. Gabelli 65, 35121 Padova, Italy

e-mail: andrea.porzionato@unipd.it
The carotid body (CB) may undergo different structural changes during perinatal development, aging, or in response to environmental stimuli. In the previous literature, morphometric approaches to evaluate these changes have considered quantitative first order parameters, such as volumes or densities, while changes in spatial disposition and/or complexity of structural components have not yet been considered. In the present study, different strategies for addressing morphological complexity of CB, apart from the overall amount of each tissue component, were evaluated and compared. In particular, we considered the spatial distribution of connective tissue in the carotid bodies of young control subjects, young opiate-related deaths and aged subjects, through analysis of dispersion (Morisita's index), gray level co-occurrence matrix (entropy, angular second moment, variance, correlation), and fractal analysis (fractal dimension, lacunarity). Opiate-related deaths and aged subjects showed a comparable increase in connective tissue with respect to young controls. However, the Morisita's index ( $p<$ $0.05)$, angular second moment $(p<0.05)$, fractal dimension $(p<0.01)$, and lacunarity $(p<0.01)$ permitted to identify significant differences in the disposition of the connective tissue between these two series. A receiver operating characteristic (ROC) curve was also calculated to evaluate the efficiency of each parameter. The fractal dimension and lacunarity, with areas under the ROC curve of 0.9651 (excellent accuracy) and 0.8835 (good accuracy), respectively, showed the highest discriminatory power. They evidenced higher level of structural complexity in the carotid bodies of opiate-related deaths than old controls, due to more complex branching of intralobular connective tissue. Further analyses will have to consider the suitability of these approaches to address other morphological features of the $\mathrm{CB}$, such as different cell populations, vascularization, and innervation.

Keywords: morphometry, fractal parameters, co-occurrence matrix, Morisita's index, carotid body, drug-related death, heroin, aging

\section{INTRODUCTION}

The carotid body $(\mathrm{CB})$ is the main peripheral arterial chemoreceptor, sensitive to reduction in $\mathrm{pO}_{2}$ and $\mathrm{pH}$ and to increases in $\mathrm{pCO}_{2}$. From a structural point of view, it is composed of lobules containing type I cells, positive for tyrosine hydroxylase, and type II cells, positive for glial fibrillary acidic protein. Type I cells are considered the true chemoreceptor elements. They are roundish and produce many different neurotransmitters and peptide neuromodulators. Type II cells are fusiform and envelop clusters of type I cells. They are usually considered supportive cells, although they may also be stem cell precursors for type I cells (Pardal et al., 2007; Platero-Luengo et al., 2014) and probably co-ordinate chemosensory transduction through interactions with the other cells of the CB (Tse et al., 2012). Connective tissue also characterizes the $\mathrm{CB}$ structure, mainly delimiting the glomic lobules (interlobular connective tissue) and partly branching in the lobular context (intralobular connective tissue). Neurotransmitters and neuromodulators released by type I cells mainly act on the afferent endings of the carotid sinus nerve, arising from the glossopharyngeal nerve. The $\mathrm{CB}$ also shows sensory innervation from jugular and nodose ganglia, post-ganglionic sympathetic nerve fibers from the superior cervical ganglion, and preganglionic parasympathetic and sympathetic fibers reaching local ganglion cells. Moreover, the CB is the structure in the body with the highest blood flow (Daly et al., 1954; Barnett et al., 1988) and local changes in blood flow have been considered to be involved in CB chemoreceptor discharge (Joels and Neil, 1963; Kirby and McQueen, 1984; Porzionato et al., 2006, 2011a,b).

The CB undergoes structural and functional changes during perinatal development (e.g., Porzionato et al., 2008a,b; De Caro et al., 2013), aging (e.g., Di Giulio et al., 2009, 2012; Zara et al., 2013a) and in response to a variety of environmental stimuli, such as chronic sustained hypoxia (e.g., Pardal et al., 2007; Platero-Luengo et al., 2014), chronic intermittent hypoxia (e.g., Iturriaga et al., 2009), chronic hyperoxia (e.g., Bavis et al., 2013), and exposure to nicotine (e.g., Stéphan-Blanchard et al., 2013). Several morphometrical approaches have been involved to address structural changes in the CB. Most morphometrical 
parameters addressed in the literature are first order parameters, such as volumes or densities. Volume analyses may involve CB in toto or its different components (parenchyma, interlobular or intralobular connective tissue, vessels) (e.g., Dinsdale et al., 1977; Lack et al., 1986; Clarke et al., 2000; Porzionato et al., 2005). Innervation of the CB has mainly been evaluated in terms of density values (e.g., Kusakabe et al., 2003, 2004). The different cell types of the CB (type I and II cells, progenitors, macrophages, mast cells, and other immune cells) have been considered in the literature in terms of cell densities or total cell numbers (e.g., Pardal et al., 2007; Porzionato et al., 2013). Computerassisted image analysis of protein expression in immunostained sections has also been performed through quantification of the immunoreactive area in order to estimate the percentage of tissue exhibiting positivity (e.g., Di Giulio et al., 2012; Zara et al., 2013b).

Size parameters alone, however, may be inadequate to fully characterize the microarchitecture generated by tissue components such as connective tissue, type II cells, vessels, and innervation, all characterized by a quite complex spatial arrangement. In fact, a pattern of this type, for each given size, can generate in the available space substantially different spatial textures characterized by different degrees of homogeneity and morphological complexity (Guidolin et al., 2004a,b). In the present study possible strategies to morphometrically estimate these morphological features of the CB tissue have been considered. In particular, they will be used for the analysis of the pattern of fibrosis induced in the CB by normal aging and opiate abuse in young people. Since data exist showing that a comparable increase in the amount of connective tissue in the $\mathrm{CB}$ occurs in both conditions, but with a likely different pattern of spatial distribution (Porzionato et al., 2005), this specific example can allow a test of the efficiency of the considered methods.

\section{MATERIALS AND METHODS TISSUE SAMPLES}

Materials consisted of carotid bodies obtained at autopsy from 35 subjects who died of heroin/morphine intoxication (26 males, nine females; mean age $( \pm S D) 26 \pm 3.5$ years). In all cases, there was a clinical history of at least 3 years of heroin addiction. The other two groups for comparison consisted of 10 young (five males, five females; mean age $22 \pm 3.4$ years) and 10 aged subjects (five males, five females; mean age $66.5 \pm 3.5$ years) who died of trauma. All subjects were clinically without chronic pulmonary or cardiovascular disease. Cardiac hypertrophy or preceding myocardial infarction were excluded at autopsy. Autopsies were performed between 24 and $78 \mathrm{~h}$ after death. Specimens were taken of the right carotid bifurcation, including $20 \mathrm{~mm}$ of the common carotid and $20 \mathrm{~mm}$ of the internal and external carotid arteries.

Autopsies and all the procedures applied to process samples from human tissues have been performed according to the Italian Mortuary Police Legislation.

\section{HISTOLOGICAL TECHNIQUES}

Tissues were fixed in 10\% phosphate-buffered formalin for $72 \mathrm{~h}$, dehydrated through ascending alcohols and xylene, and paraffin embedded. Longitudinal serial sections, $5 \mu \mathrm{m}$ thick, of the whole carotid bifurcation were then obtained, de-waxed (xylene and alcohol progressively at lower concentrations), and stained with Azan-Mallory (AM).

\section{IMAGE ANALYSIS PROCEDURES}

All the image analysis procedures were performed by using the Image J software (Schneider et al., 2012), freely available at http:// rsb.info.nih.gov/ij/. They can be summarized as follows.

\section{Image acquisition and pre-processing}

Bright-field images of the AM-stained preparations were acquired by using a Leica DMR microscope (Leica Microsystems, Wetzlar, Germany) and a high resolution digital camera (DC 200, Leica Microsystems). At a primary magnification of $\times 20$ one field per section, randomly chosen within the $\mathrm{CB}$ tissue, was selected and its image acquired in full colors (RGB, 24-bit), processed to correct shading, then filed TIFF (Figure 1A).

Since it showed the best contrast between the connective tissue and the CB parenchima, the red component of each acquired RGB image was selected for further processing (Figure 1B). Stromal structures and filaments can be easily segmented with conventional thresholding methods, and small remaining artifacts can be removed from the resulting binary image by applying a geometric filter to eliminate profiles within a specified range of area and/or shape (see Russ, 2011), leading to the generation of binary images (Figure 1C) of the connective component.

The amount of CB tissue it accounts for can be directly estimated from the corresponding binary image by evaluating the area fraction occupied by the binary pattern (Russ and Dehoff, 2000).

\section{Morphological complexity of the pattern of the connective tissue}

The obtained gray-level and binary images illustrated in Figure 1, however, can also represent the input data for procedures aimed at estimating indices able to capture more detailed morphological features, such as a characterization of the overall shape of the patterns generated by connective tissue, and of the way it arranges itself in the tissue. In these respect, three methods were considered in the present study. They are briefly detailed in the sections that follow.

Analysis of dispersion. To provide a quantitative evaluation of the dispersion in the tissue space of the binary pattern corresponding to connective tissue, Morisita's index (Morisita, 1962), one of the most robust distribution measures (Myers, 1978), was estimated. For this purpose, the binary image was divided into 12 sub-images and the number of pattern pixels in each sub-picture was evaluated. The index of dispersal $\left(I_{d}\right)$ was then calculated using

$$
I_{d}=n\left(\frac{\sum_{i=1}^{n} X_{i}^{2}-N}{N(N-1)}\right)
$$

where $n$ is the number of sub-images, whereas $N$ and $X_{i}$ represent the number of pattern pixels in the image, and in each sub-image, respectively. The index value increases with increasing spatial dispersion of the pattern. 


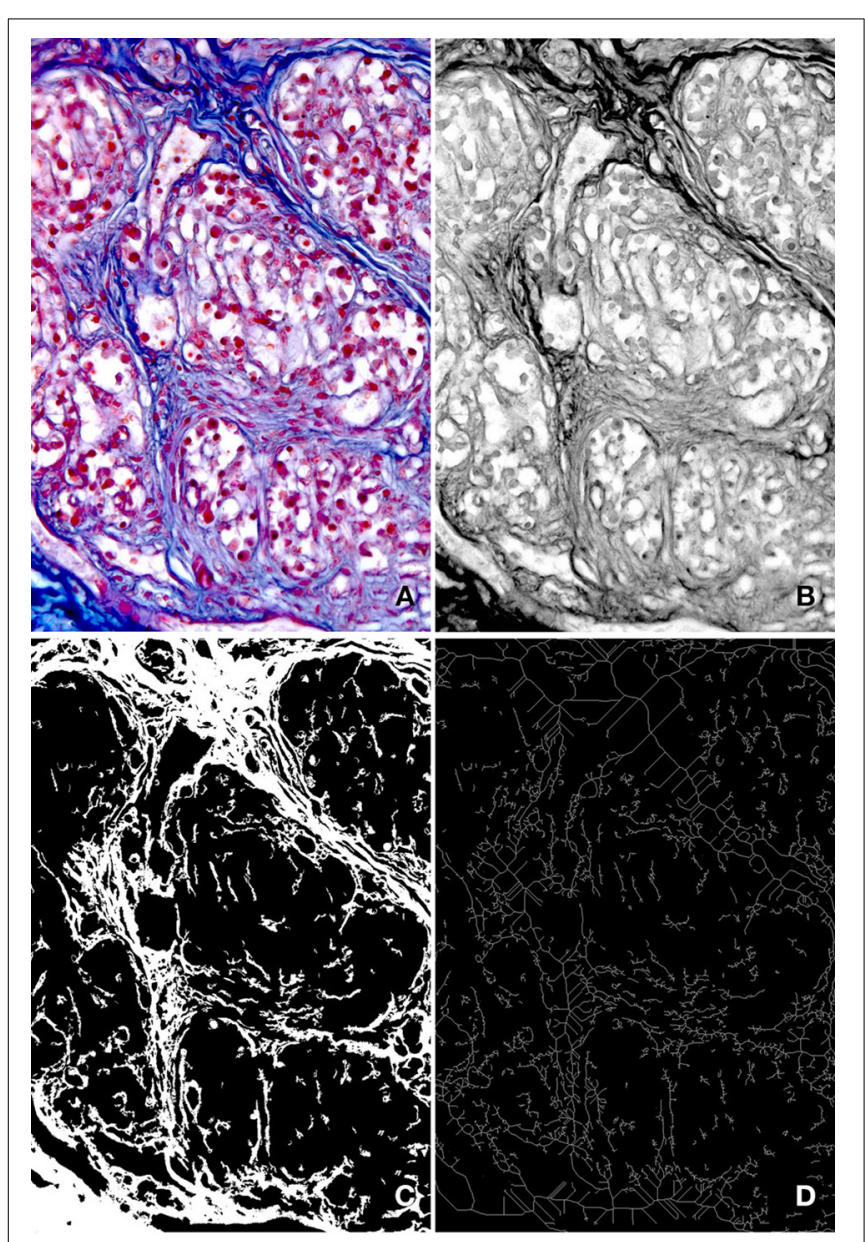

FIGURE 1 | Main steps of the image analysis procedure. (A) Full color (RGB, 24-bit) digital image of a microscope field stained with AM (primary magnification $\times 20$ ). (B) Gray level image corresponding to the red component of the image in (A). Due to the high contrast between connective tissue and parenchyma it exhibits, it was used to estimate the GLCM and to discriminate the connective tissue by proper thresholding. (C) Binary image of the connective tissue pattern, used to estimate the percent area it occupies and the Morisita's index. (D) Binary skeleton of the image in (C), from which fractal parameters were estimated.

Gray level co-occurrence matrix analysis. Gray level cooccurrence matrix (GLCM) is a fast mathematical method for assessing image structural properties such as homogeneity, complexity, and level of disorder (see Pantic et al., 2012). It was first introduced by Haralick et al. (1973) and is based on a quantitation of the relationship between pixel brightness values in an image. This information can be extracted from a matrix $P_{\theta d}(i, j)$ (GLCM) describing how frequently two pixels with gray level $i$ and $j$ appear in the image separated by a distance $d$ in the direction $\theta$ (Aggarwal and Agrawal, 2012). Haralick et al. (1973) described 14 parameters that can be calculated from the GLCM with the intent of describing the texture of an image. Today, however, those proven as the most useful in experimental and clinical medicine applications (see Losa and Castelli, 2005; Alvarenga et al., 2010; Pantic et al., 2012) are the following ones:

$$
\begin{array}{r}
\text { Entropy }=-\sum_{i} \sum_{j} P(i, j) \log (P(i, j)) \\
\text { Angular second moment }=\sum_{i} \sum_{j}[P(i, j)]^{2} \\
\text { Variance }=\sum_{i} \sum_{j}(1-\mu)^{2} P(i, j) \\
\text { Correlation }=\frac{\sum_{i} \sum_{j} i j P(i, j)-\mu_{x} \mu_{y}}{\sigma_{x} \sigma_{y}}
\end{array}
$$

where $i$ and $j$ are coordinates of the co-occurrence matrix, $\sigma^{\prime} s$ and $\mu^{\prime} s$ represent means and standard deviations along rows and columns of the matrix. They were computed with ImageJ by using the "texture analysis" plugin, developed by Julio E. Cabrera and Toby C. Cornish, and freely available at http://rsbweb.nih.gov/ij/ plugins/texture.html.

Fractal analysis. To globally describe the complexity of form in quantitative terms the "Fractal dimension" (D) can be a valuable parameter (Guidolin et al., 2004b). It measures the rate of addition of structural detail with increasing magnification, scale, or resolution (Cutting and Garvin, 1987). D of the binary skeleton (Figure 1D) was estimated using the "box counting" method at multiple origins as indicated by Smith et al. (1996). Briefly, from grids of increasing size overlying the image, the number of boxes containing any pixel was counted. This number was recorded as a function of grid size and D was calculated, as -1 times the slope of the regression line, from a plot of the log of size on the $\mathrm{x}$-axis and the log of box count on the $y$-axis. To minimize grid location effects, the algorithm started from a number (10 in our case) of locations, generating a set of values for $\mathrm{D}$. The average value over all locations was considered as the final estimate of D. During the same analytical process "Lacunarity" was also calculated. This parameter is a measure of the nonuniformity (heterogeneity) of structure or the degree of structural variance within an object (Smith et al., 1996). It was estimated as the average of the coefficient of variation for pixel density over all grid sizes and locations (Bassinghtwaighte et al., 1994).

To perform the abovementioned analysis, the "FracLac for ImageJ" plugin by Audrey Karperien was used (freely available at http://rsb.info.nih.gov/ij/plugins/fraclac/fraclac.html).

\section{Statistical analysis}

Statistical analysis was done using GraphPad Prism software (GraphPad Inc., La Jolla, CA, USA) and SPSS statistical package (v. 13.0; IBM, Armonk, NY, USA). Data were analyzed by OneWay analysis of variance followed by Dunnet's test for multiple comparisons vs. the young control group. Bonferroni's test for comparisons between selected groups was used to determine possible statistically significant differences between opiate-addicted and aged cases. $p<0.05$ was always used as the limit for statistical significance. In addition to the standard statistical difference tests, the ability of the various parameters to discriminate between aging and opiate addiction was estimated by calculating the ROC curves (Metz, 1978). ROC curve is constructed based on the fraction of true positives out of the positives (sensitivity) and the 
fraction of false positives out of the negatives (specificity), taking into account different thresholds. The potential discriminatory performance of each parameter can be estimated by analyzing the area under its ROC curve (see Zweig and Campbell, 1993). A parameter with no discriminative value would have the area under the ROC curve approximately higher than 0.5 and lower than 0.6. Area between 0.6 and 0.7 indicates "poor" performance, area between 0.7 and 0.8 "fair" performance, and area between 0.8 and 0.9 "good" performance (Zweig and Campbell, 1993; Sandelowsky et al., 2011). Parameters belonging to the category "excellent" usually have the ROC areas higher than 0.9.

\section{RESULTS}

As shown in Figure 2 a similar, statistically significant, increase in the total amount of connective tissue was observed in the $\mathrm{CB}$ from aged subjects and from young people who died of opiate intoxication when compared to normal young CB samples. It appeared mainly located between the lobes of parenchyma. From a qualitative point of view, however, some difference between the two groups can be observed in the spatial distribution of this tissue component. In particular a more complex branching of connective tissue within the $\mathrm{CB}$ parenchyma lobes seems to characterize the samples from subjects who died of opiate abuse when compared to normal aging (Figure 3).

As shown in Table 1, almost all of the morphometric parameters estimated to characterize the spatial organization of this tissue component indicated significant differences in this tissue feature between young controls and the other two groups of patients, with the only exception of $\mathrm{D}$, that exhibited a similar value in young controls and aged subject and increased significantly only in opiate-addicted group. Interestingly, however, only some of these methods were able discriminate between aged subjects and young people who died of opiate abuse. As summarized in Table 1, "Angular second moment" of the GLCM, fractal

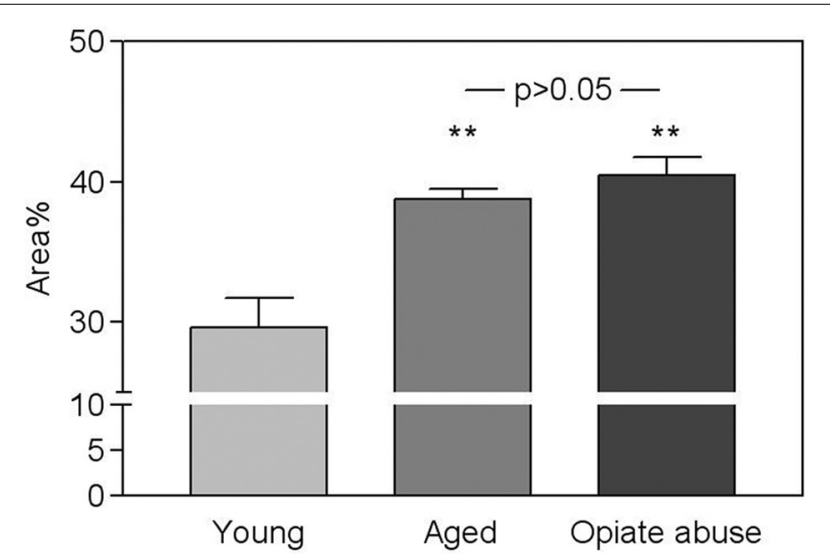

FIGURE 2 | Fraction of the CB corresponding to connective tissue in young controls, in aged subjects, and in young people who died of chronic opiate abuse. Values are average percent areas ( \pm s.e.m.). As indicated both aging and opiate abuse induced a significant increase $\left({ }^{* *} p<0.01\right)$ of the total amount of connective tissue in the CB with respect to young control subjects. Between the two conditions however, no statistically significant differences can be detected. parameters ( $\mathrm{D}$ and lacunarity), and the Morisita's index assumed significantly different values in the two conditions.

For each of these parameters a ROC curve was calculated to illustrate the efficiency of the parameter as a binary classifier of $\mathrm{CB}$ fibrosis between aged and opiate addicted subjects. As presented in Table 2, the areas under the ROC curves indicated that the fractal parameters (D and lacunarity) were those exhibiting the highest discriminatory power.

\section{DISCUSSION}

A significant increase in connective tissue and a concomitant reduction in glomic parenchyma are well known characteristics of the CB tissue in both opiate-addicted and aged cases when compared to young controls. Consistently with previously reported data (Porzionato et al., 2005), also in the present study both these conditions led to an increase of the amount of connective tissue from about $30 \%$, that can be observed in normal young people, to more than $40 \%$ of the CB. Such a change is in accordance with the findings of Hurst et al. (1985) who found progressive arteriosclerosis of the glomic arteries during aging, to which they ascribed the increase in connective tissue. Also in opiate addicts, the increase in connective tissue may be interpreted as a sign of early tissue aging, that can be ascribed to arteriosclerosis of the glomic arteries, to the recurrent episodes of hypoxia during reaction to opiate assumption, and/or to local inflammatory infiltrates (chronic carotid glomitis) (Porzionato et al., 2009).

The connective tissue, however, exhibits a complex spatial organization. It is mainly formed by large bundles of fibers located between the lobes of parenchyma, from which thin branches of intralobular connective tissue depart. When compared to young controls (see Porzionato et al., 2005), this intralobular component of the pattern appeared increased in both aged and opiate-addicted subjects. An analysis based on the simple estimate of a dimensional parameter (volume fraction) failed in detecting significant differences between the two conditions, although the branching pattern of connective tissue in opiate-addicted subjects appeared (at least qualitatively) more complex.

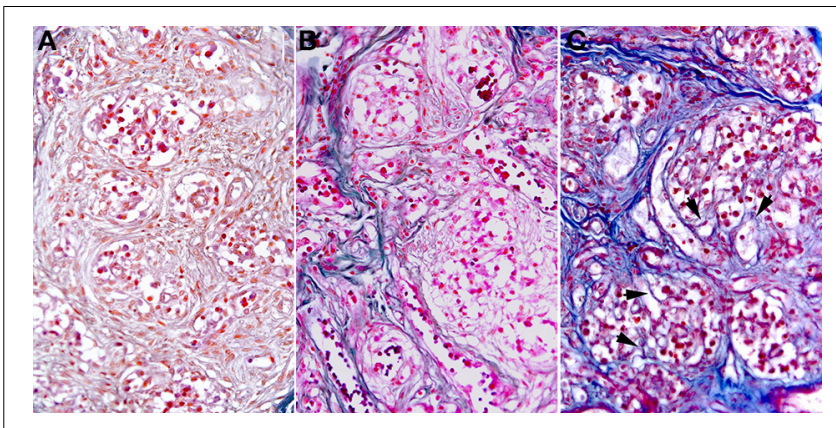

FIGURE 3 | Microscope fields of AM-stained samples from a young control (A), an aged subject (B), and from a subject who died of chronic opiate abuse (C). In the latter the spatial organization of the connective tissue appeared more complex, being characterized by a higher presence of thin branches of connective tissue within the parenchyma lobes. Some of them are highlighted by the arrow heads. 
Table 1 | Mean values ( \pm s.e.m.) of the parameters quantifying textural properties of the connective tissue in CB samples from normal young subjects, from aged subjects, and from young subjects who died of chronic opiate abuse.

\begin{tabular}{lccc}
\hline Feature & Young controls & Aging & Drug-related deaths \\
\hline Morisita's index & $0.028 \pm 0.002$ & $0.060 \pm 0.010\left({ }^{\circ}\right)$ & $0.083 \pm 0.005\left({ }^{\circ \circ},{ }^{*}\right)$ \\
Angular second moment & $0.0048 \pm 0.0006$ & $0.0020 \pm 0.0007\left(\left(^{\circ}\right)\right.$ & $0.0010 \pm 0.0001\left({ }^{\circ \circ},{ }^{*}\right)$ \\
Variance & $151.0 \pm 12.9$ & $97.2 \pm 4.8\left({ }^{\circ}\right)$ & $115.4 \pm 5.2\left({ }^{\circ}\right)$ \\
Correlation & $0.0010 \pm 0.00017$ & $0.0004 \pm 0.00005\left(\left(^{\circ}\right)\right.$ & $0.0004 \pm 0.00003\left({ }^{\circ}\right)$ \\
Entropy & $7.91 \pm 0.150$ & $8.36 \pm 0.099\left({ }^{\circ}\right)$ & $8.44 \pm 0.079\left({ }^{\circ}\right)$ \\
Fractal dimension & $1.43 \pm 0.028$ & $1.447 \pm 0.013$ & $1.563 \pm 0.006\left({ }^{\circ \circ},{ }^{* *}\right)$ \\
Lacunarity & $0.828 \pm 0.017$ & $0.500 \pm 0.021\left(^{\circ}\right)$ & $0.388 \pm 0.009\left(^{\circ \circ},{ }^{* *}\right)$
\end{tabular}

${ }^{\circ} p<0.05,{ }^{\circ} p<0.01$ vs. the "Young controls" group; ${ }^{*} p<0.05,{ }^{* *} p<0.01$ vs. the "Aging" group.

Table 2 | Area under the ROC curve for the parameters showing any discriminative power between drug-related deaths and aged subjects, and classification of their accuracy (see Zweig and Campbell, 1993; Sandelowsky et al., 2011).

\begin{tabular}{lcl}
\hline Feature & Area under the ROC curve & Accuracy \\
\hline Morisita's index & 0.6529 & Poor \\
Angular second moment & 0.7895 & Fair \\
Fractal dimension & 0.9651 & Excellent \\
Lacunarity & 0.8835 & Good \\
\hline
\end{tabular}

Thus, in the present study additional morphometric strategies were also explored, based on the analysis of the connective tissue pattern in terms of textural properties (such as homogeneity, complexity and level of disorder) more than overall amount. In particular, three different image analysis methods were tested: analysis of dispersion, analysis of the GLCM, and fractal analysis. Analysis of dispersion has been successfully used to study how a given population or morphological pattern fills the available space (see Goodenough and Goodenough, 2012). GLCM was successfully applied in nuclear magnetic resonance imaging (Li et al., 2009), computed tomography (Huber et al., 2011) and other clinical research areas. In fundamental medical and biology research, it was used to study tissue age-related structural degradation (Shamir et al., 2009), and chromatin structural changes during apoptosis (Losa and Castelli, 2005; Pantic et al., 2012). Fractal analysis proven very useful to characterize the complex morphology of vascular trees and the endothelial cells self-organization in vitro (Guidolin et al., 2004a,b). Although the interest was mainly focused on the comparison between opiate-addicted and aged cases (showing comparable amounts of connective tissue), the analysis was also extended to young cases for completeness.

The results indicated that GLCM, Morisita's index, and the fractal parameter "lacunarity" were able to discriminate normal $\mathrm{CB}$ tissue samples from those derived from aged or opiateaddicted people. However, since all the above mentioned parameters are measures of homogeneity (Morisita, 1962; Smith et al., 1996; Marrón, 2012; Pantic et al., 2013) describing how well a pattern fills the available space as a consequence of the triggering conditions, this finding could be partly related to the increase of connective tissue occurring following aging and opiate addiction.
More interesting findings emerge when conditions characterized by a similar total amount of CB connective tissue were compared. In this respect, the results of the present study indicate that some of the parameters provided by the tested methods (namely angular second moment, lacunarity, and Morisita's index) exhibited discriminatory power between $\mathrm{CB}$ normal aging and chronic opiate consumption, although in the presence of comparable amount of connective tissue. These parameters captured the qualitative observation (Porzionato et al., 2005) that following chronic opiate consumption the intralobular connective tissue branches in a more irregular way than in samples from aged subjects.

The parameter D deserves a more specific comment. Unlike the other parameters (estimating spatial homogeneity), it measures the rate of addition of structural detail with increasing magnification, scale, or resolution (Cutting and Garvin, 1987). Thus, it is particularly useful to describe in a compact form the "complexity of shape" of a structure. In this respect, the connective tissue pattern in the $\mathrm{CB}$ of opiate addicted subjects exhibited a statistically significant higher level of structural complexity when compared to the one observed in aged subjects. Also this finding is consistent with a higher degree of branching likely involving the intralobular regions of the CB. Interestingly, as far as this parameter is concerned, no significant differences were observed between aged and young subjects, showing that this parameter is not influenced (or only minimally) by the overall amount of connective tissue. Instead, the parameter D showed significantly different values between aged and opiate-addicted subjects, confirming the capability of this parameter to catch differences in the complexity of disposition of connective tissue, independently from quantitative aspects. Thus, we may state that beyond differences in the total amount, $\mathrm{CB}$ connective tissue is similarly organized in young and aged subjects whereas it is organized in a more complex irregular way in the opiate addicted subjects.

In addition to the standard statistical difference tests, the ability of the abovementioned parameters to discriminate between the two groups with comparable amount of connective tissue was here tested by ROC analysis, a technique essential in clinical sciences for evaluating the potential value of a diagnostic test (Pantic et al., 2013). According to this method, the potentially discriminatory performance of a parameter is evaluated by analyzing the area under the ROC curve. In this study the parameters provided by the fractal analysis ( $D$ and lacunarity) showed the highest accuracy and efficiency as binary classifiers. Thus, they appear as a 
particular useful tool to complement dimensional parameters in order to describe complex tissue patterns (as the one exhibited by the connective tissue in the $\mathrm{CB}$ ) and the changes they undergo under pathological conditions.

It can also be emphasized that the presented methods are not limited to the specific staining method used in the present study, but they can be applied to all the methods commonly used to visualize the connective tissue (as, for instance, Masson's trichromic and Sirius red), provided the image pre-processing includes a suitable segmentation step for this tissue component, such as color thresholding or color deconvolution (Ruifrok and Johnston, 2001; Rey et al., 2008), which must be considered preliminary to the use of the above methods. Furthermore, the analysis here presented could be easily adapted and extended to provide a morphometric description of spatial complexity of other CB tissue patterns, such as those generated by sustentacular type II cells, vessels, or innervation. In various experimental or clinical conditions, these structural components could have no changes in terms of volume fraction or density but changes in spatial disposition which could have a biological significance and which could be morphometrically described with the above methods, and particularly with fractal analysis.

\section{AUTHOR CONTRIBUTIONS}

All the authors (Diego Guidolin, Andrea Porzionato, Cinzia Tortorella, Veronica Macchi, and Raffaele De Caro) contributed substantially to the conception or design of the work, or to the acquisition, analysis, or interpretation of data of the work. All the authors drafted or revised the work critically for important intellectual content, and they approved the final version to be published. All the authors agree to be accountable for all aspects of the work in ensuring that questions related to the accuracy or integrity of any part of the work are appropriately investigated and resolved.

\section{ACKNOWLEDGMENT}

The study was supported by a grant from the University of Padova (ex-60\%) to Cinzia Tortorella.

\section{REFERENCES}

Aggarwal, N., and Agrawal, R. K. (2012). First and second order statistics features for classification of magnetic resonance brain images. J. Signal Inform. Process. 3, 146-153. doi: 10.4236/jsip.2012.32019

Alvarenga, A. V., Teixeira, C. A., Ruano, M. G., and Pereira, W. C. (2010). Influence of temperature variations on the entropy and correlation of the greylevel co-occurrence matrix from b-mode images. Ultrasonics 50, 290-293. doi: 10.1016/j.ultras.2009.09.002

Barnett, S., Mulligan, E., Wagerle, L. C., and Lahiri, S. (1988). Measurement of carotid body blood flow in cats by use of radioactive microspheres. J. Appl. Physiol. 65, 2484-2489.

Bassinghtwaighte, J. B., Leibovitch, L. S., and West, B. J. (1994). Fractal Physiology. American Physiological Society Methods in Physiology Series. New York, NY: Oxford University Press.

Bavis, R. W., Fallon, S. C., and Dmitrieff, E. F. (2013). Chronic hyperoxia and the development of the carotid body. Respir. Physiol. Neurobiol. 185, 94-104. doi: 10.1016/j.resp.2012.05.019

Clarke, J. A., Daly, M. B., Marshall, J. M., Ead, H. W., and Hennessy, E. M. (2000). Quantitative studies of the vasculature of the carotid body in the chronically hypoxic rat. Braz. J. Med. Biol. Res. 33, 331-340. doi: 10.1590/S0100879X2000000300012
Cutting, J. E., and Garvin, J. J. (1987). Fractal curves and complexity. Percept. Psychophysiol. 42, 365-370. doi: 10.3758/BF03203093

Daly, M. B., Lambertsen, C. J., and Schweitzer, A. (1954). Observations on the volume of blood flow and oxygen utilization of the carotid body in the cat. J. Physiol. 125, 67-89.

De Caro, R., Macchi, V., Sfriso, M. M., and Porzionato, A. (2013). Structural and neurochemical changes in the maturation of the carotid body. Respir. Physiol. Neurobiol. 185, 9-19. doi: 10.1016/j.resp.2012.06.012

Di Giulio, C., Antosiewicz, J., Walski, M., Petruccelli, G., Verratti, V., Bianchi, G., et al. (2009). Physiological carotid body denervation during aging. Adv. Exp. Med. Biol. 648, 257-263. doi: 10.1007/978-90-481-2259-2_29

Di Giulio, C., Zara, S., Cataldi, A., Porzionato, A., Pokorski, M., and De Caro, R. (2012). Human carotid body HIF and NGB expression during human development and aging. Adv. Exp. Med. Biol. 758, 265-271. doi: 10.1007/97894-007-4584-1_36

Dinsdale, F., Emery, J. L., and Gadsdon, D. R. (1977). The carotid body - a quantitative assessment in children. Histopathology 1, 179-187. doi: 10.1111/j.13652559.1977.tb01657.x

Goodenough, A. E., and Goodenough, A. S. (2012). Development of a rapid and precise method of digital image analysis to quantify canopy density and structural complexity. ISRN Ecol. 2012:619842. doi: 10.5402/2012/619842

Guidolin, D., Nico, B., Mazzocchi, G., Vacca, A., Nussdorfer, G. G., and Ribatti, D. (2004a). Order and disorder in the vascular network. Leukemia 18, 1745-1750. doi: 10.1038/sj.leu.2403526

Guidolin, D., Vacca, A., Nussdorfer, G. G., and Ribatti, D. (2004b). A new image analysis method based on topological and fractal parameters to evaluate the angiostatic activity of docexatel by using the Matrigel assay in vitro. Microvasc. Res. 67, 117-124. doi: 10.1016/j.mvr.2003.11.002

Haralick, R. M., Hudson, L. L., and Dinstein, I. (1973). Textural features for image classification. IEEE Trans. Syst. Man Cybern. 3, 610-621. doi: 10.1109/TSMC.1973.4309314

Huber, M. B., Nagarajan, M. B., Leinsinger, G., Eibel, R., Ray, L. A., and Wismüller, A. (2011). Performance of topological texture features to classify fibrotic interstitial lung disease patterns. Med. Phys. 38, 2035-2044. doi: 10.1118/1.3566070

Hurst, G., Hearth, D., and Smith, P. (1985). Histological changes associated with ageing of the human carotid body. J. Pathol. 147, 181-187. doi: 10.1002/path.1711470306

Iturriaga, R., Moya, E. A., and Del Rio, R. (2009). Carotid body potentiation induced by intermittent hypoxia: implications for cardiorespiratory changes induced by sleep apnoea. Clin. Exp. Pharmacol. Physiol. 36, 1197-1204. doi: 10.1111/j.1440-1681.2009.05213.x

Joels, N., and Neil, E. (1963). The excitation mechanisms of the carotid body. $\mathrm{Br}$. Med. Bull. 19, 21-24.

Kirby, G. C., and McQueen, D. S. (1984). Effects of the antagonists MDL 7222 and ketanserin on responses of cat carotid body chemoreceptors to 5-hydroxytryptamine. Br. J. Pharmacol. 83, 259-269. doi: 10.1111/j.14765381.1984.tb10142.x

Kusakabe, T., Hirakawa, H., Matsuda, H., Kawakami, T., Takenaka, T., and Hayashida, Y. (2003). Peptidergic innervation in the rat carotid body after 2, 4, and 8 weeks of hypocapnic hypoxic exposure. Histol. Histopathol. 18, 409-418.

Kusakabe, T., Hirakawa, H., Oikawa, S., Matsuda, H., Kawakami, T., Takenaka, T., et al. (2004). Morphological changes in the rat carotid body 1, 2, 4, and 8 weeks after the termination of chronically hypocapnic hypoxia. Histol. Histopathol. 19, 1133-1140.

Lack, E. E., Perez-Atayde, A. R., and Young, J. B. (1986). Carotid bodies in sudden infant death syndrome: a combined light microscopic, ultrastructural, and biochemical study. Pediatr. Pathol. 6, 335-350. doi: 10.3109/15513818609037724

Li, X., Pai, A., Blumenkrantz, G., Carballido-Gamio, J., Link, T., Ma, B., et al. (2009). Spatial distribution and relationship of T1rho and T2 relaxation times in knee cartilage with osteoarthritis. Magn. Reson. Med. 61, 1310-1318. doi: $10.1002 / \mathrm{mrm} .21877$

Losa, G. A., and Castelli, C. (2005). Nuclear patterns of human breast cancer cells during apoptosis: characterisation by fractal dimension and co-occurrence matrix statistics. Cell Tissue Res. 322, 257-267. doi: 10.1007/s00441-005-0030-2

Marrón, B. (2012). Co-occurrence matrix and fractal dimension for image segmentation. Rev. Mate. Teor. Aplic. 19, 49-63.

Metz, C. E. (1978). Basic principles of ROC analysis. Semin. Nucl. Med. 8, 283-298. doi: 10.1016/S0001-2998(78)80014-2 
Morisita, M. (1962). $I_{d}$-index, a measure of dispersion of individuals. Res. Popul. Ecol. 4, 1-7. doi: 10.1007/BF02533903

Myers, J. H. (1978). Selecting a measure of dispersion. Environ. Entomol. 7, 619-621.

Pantic, I., Pantic, S., and Basta-Jovanovic, G. (2012). Grey level co-occurrence matrix (GLCM) texture analysis of germinal center light zone lymphocyte nuclei: physiology viewpoint with focus on apoptosis. Microsc. Microanal. 18, 470-475. doi: $10.1017 /$ S1431927612000098

Pantic, I., Pantic, S., Paunovic, J., and Perovic, M. (2013). Nuclear entropy, angular second moment, variance and texture correlation of thymus cortical and medullar lymphocytes: grey level co-occurrence matrix analysis. Ann. Braz. Acad. Sci. 85, 1063-1072. doi: 10.1590/S0001-37652013005000045

Pardal, R., Ortega-Sáenz, P., Durán, R., and López-Barneo, J. (2007). Glia-like stem cells sustain physiologic neurogenesis in the adult mammalian carotid body. Cell 131, 364-377. doi: 10.1016/j.cell.2007.07.043

Platero-Luengo, A., González-Granero, S., Durán, R., Díaz-Castro, B., Piruat, J. I., García-Verdugo, J. M., et al. (2014). An O2-sensitive glomus cell-stem cell synapse induces carotid body growth in chronic hypoxia. Cell 156, 291-303. doi: 10.1016/j.cell.2013.12.013

Porzionato, A., Fenu, G., Rucinski, M., Macchi, V., Montella, A., Malendowicz, L. K., et al. (2011b). KISS1 and KISS1R expression in the human and rat carotid body and superior cervical ganglion. Eur. J. Histochem. 55:e14. doi: 10.4081/ejh.2011.e14

Porzionato, A., Macchi, V., Belloni, A. S., Parenti, A., and De Caro, R. (2006). Adrenomedullin immunoreactivity in the human carotid body. Peptides 27, 69-73. doi: 10.1016/j.peptides.2005.07.017

Porzionato, A., Macchi, V., De Caro, R., and Di Giulio, C. (2013). Inflammatory and immunomodulatory mechanisms in the carotid body. Respir. Physiol. Neurobiol. 187, 31-40. doi: 10.1016/j.resp.2013.02.017

Porzionato, A., Macchi, V., Guidolin, D., Parenti, A., Ferrara, S. D., and De Caro, R. (2005). Histopathology of carotid body in heroin addiction. Possible chemosensitive impairment. Histopathology 46, 296-306. doi: 10.1111/j.13652559.2005.02060.x

Porzionato, A., Macchi, V., Parenti, A., and De Caro, R. (2008b). Trophic factors in the carotid body. Int. Rev. Cell. Mol. Biol. 269, 1-58. doi: 10.1016/S19376448(08)01001-0

Porzionato, A., Macchi, V., Parenti, A., and De Caro, R. (2009). Chronic carotid glomitis in heroin addiction. Histol. Histopathol. 24, 707-715.

Porzionato, A., Macchi, V., Parenti, A., Matturri, L., and De Caro, R. (2008a). Peripheral chemoreceptors: postnatal development and cytochemical findings in Sudden Infant Death Syndrome. Histol. Histopathol. 23, 351-365.

Porzionato, A., Rucinski, M., Macchi, V., Stecco, C., Castagliuolo, I., Malendowicz, L. K., et al. (2011a). Expression of leptin and leptin receptor isoforms in the rat and human carotid body. Brain Res. 1385, 56-67. doi: 10.1016/j.brainres.2011.02.028

Rey, S., Del Rio, R., and Iturriaga, R. (2008). Contribution of endothelin-1 and endothelin A and B receptors to the enhanced carotid body chemosensory responses induced by chronic intermittent hypoxia. Adv. Exp. Med. Biol. 605, 228-232. doi: 10.1007/978-0-387-73693-8_40

Ruifrok, A. C., and Johnston, D. A. (2001). Quantification of histochemical staining by color deconvolution. Anal. Quant. Cytol. Histol. 23, 291-299.
Russ, J. C. (2011). The Image Processing Handbook, 6th Edn. Boca Raton: Taylor \& Francis Group.

Russ, J. C., and Dehoff, R. T. (2000). Practical Stereology. New York, NY: Kluwer Academic/Plenum Publishers.

Sandelowsky, H., Ställberg, B., Nager, A., and Hasselström, J. (2011). The prevalence of undiagnosed chronic obstructive pulmonary disease in a primary care population with respiratory tract infections-a case finding study. BMC Fam. Pract. 12:122. doi: 10.1186/1471-2296-12-122

Schneider, C. A., Rasband, W. S., and Eliceiri, K. W. (2012). NIH Image to ImageJ: 25 years of image analysis. Nat. Methods 9, 671-675. doi: 10.1038/nmeth.2089

Shamir, L., Wolkow, C. A., and Goldberg, I. G. (2009). Quantitative measurement of aging using image texture entropy. Bioinformatics 25, 3060-3063. doi: 10.1093/bioinformatics/btp571

Smith, T. G., Lange, G. D., and Marks, W. B. (1996). Fractal methods and results in cellular morphology-dimensions, lacunarity and multifractals. J. Neurosci. Methods 69, 123-136. doi: 10.1016/S0165-0270(96)00080-5

Stéphan-Blanchard, E., Bach, V., Telliez, F., and Chardon, K. (2013). Perinatal nicotine/smoking exposure and carotid chemoreceptors during development. Respir. Physiol. Neurobiol. 185, 110-119. doi: 10.1016/j.resp.2012.06.023

Tse, A., Yan, L., Lee, A. K., and Tse, F. W. (2012). Autocrine and paracrine actions of ATP in rat carotid body. Can. J. Physiol. Pharmacol. 90, 705-711. doi: 10.1139/y2012-054

Zara, S., Pokorski, M., Cataldi, A., Porzionato, A., De Caro, R., Antosiewicz, J., et al. (2013a). Development and aging are oxygen-dependent and correlate with VEGF and NOS along life span. Adv. Exp. Med. Biol. 756, 223-228. doi: 10.1007/978-94-007-4549-0_28

Zara, S., Porzionato, A., De Colli, M., Macchi, V., Cataldi, A., De Caro, R., et al. (2013b). Human carotid body neuroglobin, vascular endothelial growth factor and inducible nitric oxide synthase expression in heroin addiction. Histol. Histopathol. 28, 903-911.

Zweig, M. H., and Campbell, G. (1993). Receiver-operating characteristic (ROC) plots: a fundamental evaluation tool in clinical medicine. Clin. Chem. 39, 561-577.

Conflict of Interest Statement: The authors declare that the research was conducted in the absence of any commercial or financial relationships that could be construed as a potential conflict of interest.

Received: 08 September 2014; accepted: 17 October 2014; published online: 05 November 2014.

Citation: Guidolin D, Porzionato A, Tortorella C, Macchi V and De Caro R (2014)

Fractal analysis of the structural complexity of the connective tissue in human carotid bodies. Front. Physiol. 5:432. doi: 10.3389/fphys.2014.00432

This article was submitted to Integrative Physiology, a section of the journal Frontiers in Physiology.

Copyright @ 2014 Guidolin, Porzionato, Tortorella, Macchi and De Caro. This is an open-access article distributed under the terms of the Creative Commons Attribution License (CC BY). The use, distribution or reproduction in other forums is permitted, provided the original author(s) or licensor are credited and that the original publication in this journal is cited, in accordance with accepted academic practice. No use, distribution or reproduction is permitted which does not comply with these terms. 